\title{
Deltoid Ligament Injury and Ankle Fractures: To Fix the Ligament or Not
}

\author{
Sharad Prabhakar ${ }^{1}$, Siddhartha Sharma ${ }^{2}$ \\ Journal of Foot and Ankle Surgery (Asia Pacific) (2020): 10.5005/jp-journals-10040-1119
}

The current journal issue has an interesting debate on methods of treating the injured deltoid ligament in association with ankle fractures. The deltoid ligament has been involved in up to $40 \%$ of ankle fractures; most of these are often due to a complex combination of sequential bony and ligamentous injuries, whose severity is dependent on foot position and the direction of deforming forces. ${ }^{1,2}$ Lauge Hansen ${ }^{1}$ clearly stated that supination external rotation (SER) injuries account for nearly $80 \%$ of ankle fractures and that there was either a deltoid ligament tear or a medial malleolus fracture in stage IV SER. In pronation-external rotation fractures $(P E R)$ involving the fibula-again there is always a fracture of the medial malleolus or a deltoid ligament tear. ${ }^{3}$ These are recognized as unstable fracture patterns and are treated operatively. ${ }^{3}$ The fibula fractures are usually treated with plate fixation. It has been suggested in the past that deltoid ligament repair is not necessary. ${ }^{4}$ The debate was regarding the necessity of deltoid ligament repair while treating an ankle fracture.

Dr Burton Dunlop and Dr Jessie Doty's opinion in the current debate outlines some of the classic studies against repair of the deltoid ligament. They mention that De Souza, ${ }^{5}$ Baird, ${ }^{6}$ Harper, ${ }^{7}$ and Zeegers et al. ${ }^{4}$ are some of the authors from the 1980 s who recommended not fixing the deltoid ligament. A subsequent randomized controlled trial (RCT) by Stromsoe et al. ${ }^{8}$ in 1995 and a meta-analysis by Dabash et al. ${ }^{9}$ in 2018 are some of the later publications quoted by them in favor of not repairing the deltoid ligament.

However, it is imperative to read between the lines. For instance, Zeegers et al. ${ }^{4}$ in their series of 290 ankle fractures have concluded that deltoid ligament fixation is not necessary. However, in that study, 28 patients were identified as having concomitant deltoid ligament injury (increased medial clear space, swelling over medial malleolus). All fractures were managed by the lateral approach only and no deltoid repair was performed. About 8 out of 28 patients (approximately 28\%) had poor results and changes of osteoarthritis at an average of 1.5 years from injury; however Zeegers et al. ${ }^{4}$ still went on to recommend against deltoid repair!

The authors also mention an old paper by Lindsjo ${ }^{10}$ from 1981, which suggested that the deltoid ligament may be injured in $10 \%$ of ankle fractures. It is pertinent to note that newer studies suggest that up to $40 \%$ of acute ankle fractures may be associated with a deltoid ligament injury; this was clearly stated by Hintermann et al. ${ }^{11}$ in the year 2000 in their series on ankle arthroscopy in 288 acute ankle fractures.

Dr Panchbhavi, in his counterpoint publication arguing for fixation of the deltoid ligament, quotes Hintermann ${ }^{11}$ as well; he is convinced that the incidence of deltoid injuries with acute ankle fractures is worth intervention. He counters the assertion made in the historical classics like the studies by De Souz ${ }^{5}$ and Stromsoe ${ }^{8}$ by stating that residual pain and deformity in those with deltoid injury was not assessed postoperatively. He also talks about elite athletes
${ }^{1,2}$ Department of Orthopedics, Postgraduate Institute of Medical Education and Research, Chandigarh, India

Corresponding Author: Siddhartha Sharma, Department of Orthopedics, Postgraduate Institute of Medical Education and Research, Chandigarh, India, Phone: +91 9988793537, e-mail: sids82@ gmail.com

How to cite this article: Prabhakar S, Sharma S. Deltoid Ligament Injury and Ankle Fractures: To Fix the Ligament or Not. J Foot Ankle Surg (Asia Pacific) 2020;7(1):8-9.

Source of support: Nil

Conflict of interest: None

who sustain high-energy trauma during sports. He mentioned that $\mathrm{Hsu}$ et al. ${ }^{12}$ observed that the deltoid complex impinges in the medial gutter or retracts distally with high-energy unstable ankle fractures. They reviewed their results in $14 \mathrm{NFL}$ players who underwent ankle fracture fixation with open deltoid complex repairs, and stated that all NFL players were able to return to running and cutting maneuvers by 6 months after surgery.

Dr Panchbhavi's paper becomes more relevant as it mentions more recent studies, specifically those by Woo et al. ${ }^{13}$ and Zhao et al..$^{14}$ these authors are in favor of deltoid ligament repair. Zhao's paper $^{14}$ concluded that surgical repair of the deltoid ligament is helpful in decreasing the postoperative medial clear space and malreduction rate, especially for the Weber $C$ ankle fractures. There is a recent meta-analysis by Salameh et al. ${ }^{15}$ that looks at primary deltoid repair in ankle fractures. The analysis included a total of 192 patients, 81 in the deltoid ligament repair group and 111 in the nonrepair group. The authors concluded that those who had their deltoid ligament repaired had superior radiological correction of the medial clear space with better pain scores.

As editors our take on the debate is as follows: most surgeons recommend deltoid fixation only if the torn ligament does not allow for adequate fracture fixation. However, in Weber B and C fractures, it is a good idea to perform an intraoperative talus stress view after fixing the fibula. If there is residual increase in medial clear space or talar tilt in the intraoperative post-fixation views, then the deltoid ligament must be fixed. ${ }^{3}$

Another subset of patients are those with a medial malleolus fracture (SER stage 4); about $25 \%$ also have disruption of the deep deltoid ligament. ${ }^{16}$ Therefore, fixation of a medial malleolus fracture with only a screw or a plate without repairing the injured deltoid ligament may not restore ankle joint stability. ${ }^{4}$ Tornetta ${ }^{17}$ showed that $26 \%$ of all patients with a fixed medial malleolar fracture had an evident incompetence of the deltoid ligament, seen radiographically as widening of the medial clear space even after fixation. Again, in such a scenario, repair of the medial deltoid ligament is warranted. This also underscores the importance of 
intraoperative stress views after bony fixation. Jones and Nunley ${ }^{18}$ have also stressed that bimalleolar-equivalent fractures (ankle fractures with deltoid ligament injury and lateral malleolus fracture) do well with deltoid ligament fixation.

In conclusion, deltoid ligament repair should be performed:

- If the talus cannot be reduced intraoperatively and the medial clear space remains wide.

- If intraoperative radiographs confirm increased medial clear space or talar tilt on talar stress views even after fibular fixation.

- Even when there is bony injury to the medial malleolus, an intra-op stress view after fixing the medial malleolus is imperative. Widening of the clear space or talar tilt warrants deltoid ligament repair.

- In Bimalleolar-equivalent fractures even after syndesmotic fixation if intraoperative stress views reveal persistent medial instability.

- In high-energy trauma in professional athletes.

\section{References}

1. Lauge-Hansen N. Ligamentous ankle fractures; diagnosis and treatment. Acta Chir Scand 1949;97(6):544-550.

2. Hintermann B, Boss A, Schafer D. Arthroscopic findings in patients with chronic ankle instability. Am J Sports Med 2002;30(3):402-409. DOI: $10.1177 / 03635465020300031601$.

3. Lötscher P, Lang TH, Zwicky L, et al. Osteoligamentous injuries of the medial ankle joint. Eur J Trauma Emerg Surg 2015;41(6):615-621. DOI: 10.1007/s00068-015-0548-2.

4. Zeegers $A V$, van der Werken $C$. Rupture of the deltoid ligament in ankle fractures: should it be repaired? Injury. 1989;20(1):39-41. DOI: 10.1016/0020-1383(89)90043-0.

5. De Souza LJ, Gustilo RB, Meyer TJ. Results of operative treatment of displaced external rotation-abduction fractures of the ankle. J Bone Joint Surg Am 1985;67(7):1066-1074. DOI: 10.2106/00004623198567070-00010.

6. Baird R, Jackson S. Fractures of the distal part of the fibule with associated disruption of the deltoid ligament. treatment without repair of the deltoid ligament. J Bone Joint Surg Am 1987;69(9):13461352. DOI: $10.2106 / 00004623-198769090-00007$.

7. Harper M. The deltoid ligament. An evaluation of need for surgical repair. Clin Orthop Relat Res 1988;226:156-168.

8. Stromsoe K, Hogevold HE, Skjeldal S, et al. The repair of a ruptured deltoid ligament is not necessary in ankle fracture. J Bone Joint Surg Br 1995;77(6):920-921. DOI: 10.1302/0301-620X.77B6.7593106.

9. Dabash S, Elabd A, Potter E, et al. Adding deltoid ligament repair in ankle fracture treatment: Is it necessary? A systematic review. Foot Ankle Surg 2018.

10. Lindsjo U. Operative treatment of ankle fractures. Acta Orthop Scand Suppl 1981;189:1-131. DOI: 10.3109/ort.1981.52.suppl-189.01.

11. Hintermann B, Regazzoni P, Lampert C, et al. Arthroscopic findings in acute fractures of the ankle. J Bone Joint Surg Br 2000;82(3):345-351. DOI: 10.1302/0301-620X.82B3.0820345.

12. Hsu AR, Lareau CR, Anderson RB. Repair of Acute superficial deltoid complex avulsion during ankle fracture fixation in National Football League players. Foot Ankle Int 2015;36(11):1272-1278. DOI: $10.1177 / 1071100715593374$.

13. Woo SH, Bae SY, Chung HJ. Short-term results of a ruptured deltoid ligament repair during an acute ankle fracture fixation. Foot Ankle Int 2018;39(1):35-45. DOI: 10.1177/1071100717732383.

14. Zhao HM, Lu J, Zhang F, et al. Surgical treatment of ankle fracture with or without deltoid ligament repair: a comparative study. BMC Musculoskelet Disord 2017;18:543. DOI: 10.1186/s12891-017-1907-4.

15. Salameh M, Alhammoud A, Alkhatib N, et al. Outcome of primary deltoid ligament repair in acute ankle fractures: a meta-analysis of comparative studies. Int Orthop 2020;44(2):341-347. DOI: 10.1007/ s00264-019-04416-9.

16. Schuberth JM, Collman DR, Rush SM, et al. Deltoid ligament integrity in lateral malleolar fractures: a comparative analysis of arthroscopic and radiographic assessments. J Foot Ankle Surg 2004;43(1):20-29. DOI: 10.1053/j.jfas.2003.11.005.

17. Tornetta 3rd P. Competence of the deltoid ligament in bimalleolar ankle fractures after medial malleolar fixation. J Bone Joint Surg Am 2000;82(6):843-848. DOI: 10.2106/00004623-200006000-00011.

18. Jones CR, Nunley 2 nd JA. Deltoid ligament repair versus syndesmotic fixation in bimalleolar equivalent ankle fractures. J Orthop Trauma 2015;29(5):245-249. DOI: 10.1097/BOT.0000000000000220. 